\title{
A REEARK ON GOLOBACH'S PROBLEM II
}

\author{
By
}

\section{S. SRINIVASAN}

For $\mathbf{A}>0$, even integer $N(>1)$, set

$$
E_{A}(N)=\sum_{q \leqslant(\log N)} A \frac{\mu^{2}(q) c_{q}(-N)}{\phi^{2}(q ;}
$$

Here, $c_{q}(n)$ is the Ramanujan's sum; one evaluation is

$$
\mathrm{c}_{\mathrm{q}}(-\mathrm{N})=\frac{\mu(\mathrm{q} /(\mathrm{q}, \mathrm{N})) \phi(\mathrm{q})}{\phi(\mathrm{q} /(\mathrm{q}, \mathrm{N}))}
$$

R. Balasubramanian and C. J. Mozzochi proved (cf. $\S 6$ of [1]) the following theorem.

Theorem.

For any fixed $A(>0)$, the relation

$$
E_{A}(N) \sim E_{\infty}(N), N \rightarrow \infty,
$$

is false.

Now we give an alternative proof of this theorem based on

(3) $\mathrm{E}_{\infty}(\mathrm{N})-\mathrm{E}_{\mathrm{A}}(\mathrm{N})=O\left(\mathrm{E}_{\infty}(\mathrm{N}) / \mathrm{A}\right), \mathrm{E}_{\infty}(\mathrm{N}) \asymp \mathrm{N} / \phi(\mathrm{N})$,

proved in [2].

\section{Remark.}

In view of the above theorem, it is necessary to state the Theorem in [2] with the factor $N / \phi(N)$ in the hypothesis; i. e., the bound

$$
\delta_{B} \frac{N}{(\log N)^{2}} \text { replaced by } \delta_{B} \frac{N}{(\log N)^{2}} \frac{N}{\phi(N)} \text {. }
$$


This can be done with no change in the proof there.

We require also the following

Lemma.

Lot $\alpha>0$. Then, as $x \rightarrow \infty$,

$\left|\left\{\mathrm{n}<\mathrm{x}: \mu(\mathrm{n}) \neq 0, \quad \mathrm{p} \mid \mathrm{n} \Rightarrow \mathrm{p} \leqslant \mathrm{x}^{a}\right\}\right|>\mathrm{c}_{a} \mathrm{x}$,

holds with some $c_{\alpha}>0$.

\section{Romark}

Actually, the above number has a well-known asymptotic formula. However, we give here a direct simple proof of this Lemma, as it is sufficient for our present purpose.

\section{Proof of the Lemma}

Clearly, it suffices to prove the Lemma for a sequence $\alpha=\alpha_{k} \rightarrow 0($ as $k \rightarrow \infty)$. We choose $\alpha_{k}=3 /(5 k+4)$; $k=1,2, \ldots$ Consider $0<\frac{2}{3} \alpha_{k}=\delta_{0}<\ldots<\delta_{R}=\alpha_{k}$ with $\mathbf{R}=2 \mathrm{k}+1 ; \theta$ defined through $\boldsymbol{\delta}_{\mathbf{j}}=\delta_{0}+\theta j$ $(\mathrm{j}=0,1, \ldots, \mathrm{R})$.

\section{Note that}

$$
\begin{aligned}
\delta_{1}+\cdots+\delta_{R-1} & =(R-1) \delta_{0}+\frac{R-1}{2} R_{\theta} \\
& =\frac{R-1}{2}\left(2 \delta_{0}+\delta_{R}-\delta_{0}\right),
\end{aligned}
$$

and on inserting the values of $\mathbf{R}$ and ${ }_{\gamma_{R}}$ in torms of $k$ we see that

$$
1-2 \delta_{0}=\delta_{1}+\ldots+\delta_{R-1}<1-\left(\delta_{R}+\delta\right)
$$

with any flsed $(0<) \delta<\frac{1}{2} \delta_{0}$.

Let $q \star$ denote a typical product $\underset{j=1}{\mathbf{R}} p_{j}$ with primes $p_{j}$ 
satisfylng $x^{\delta_{j-1}}<p_{j}<x^{\delta_{j}}$. Now, from (4), $x^{1-\delta}>q^{\star}>x^{1-\delta} 0$. Letting $q^{\prime}<x / q^{\star}$ run through square-free values, we see that $q^{\star} q^{\prime}<x$ are distinct square-free numbers, and their number is

$$
\gg \sum \frac{x}{q^{*}}=x \underset{j=1}{R}\left(\sum \frac{1}{p_{j}}\right)>c_{k}{ }^{\prime} x
$$

with some $\mathrm{c}_{\mathrm{k}}^{\prime}>0$. This completes the proof of the Lemma.

\section{Proof of the Theorem.}

In view of (3), it is sufficient to consider a lower bound for

$$
E_{A, A^{\prime}} \quad(N)=E_{A^{\prime}}(N)-E_{A^{(N)}}
$$

with a suitably large $\mathrm{A}^{\prime}(>2 \mathrm{~A}$, say). Now, we restrict $N$ to the sequence

$$
\mathrm{N}_{\mathrm{m}}=\underset{\mathrm{p}<\log \mathrm{m}}{\pi} \mathrm{p}, \quad\left(\log \mathrm{N}_{\mathrm{m}} \sim \log \mathrm{m}\right)
$$

and note that if a square-free $q \times N$, then $q /(q, N) \gg \log N$. Thus, by (1), we see the contribution of $\mathrm{q} \times \mathbf{N}$ to $\mathrm{E}_{\mathbf{A}, \mathbf{A}^{\prime}}(\mathbf{N})$ is

$$
O\left(\sum^{\prime} \frac{\mu^{2}(q)}{\phi(q)} \frac{1}{\sqrt{ } \log N}\right), \text { say, }
$$

where ' denotes the restriction on $q$ in (3'). So this contribution is

$$
O_{\mathrm{A} . \mathrm{A}^{\prime}}(\log \log \mathrm{N} / \vee \log \mathrm{N})=\mathrm{o}\left(\mathrm{E}_{\infty} \text { (N)), } \mathrm{N} \rightarrow \infty\right. \text {, }
$$

since, for $N$ in (5), $E_{x}(N) \asymp \log \log N$, by (3). Next, the

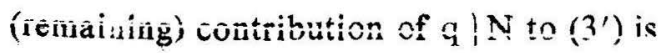




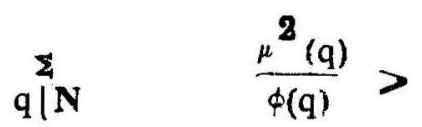

$(\log N)^{A}<q<(\log N)^{A^{\prime}}$

$$
(\log N)^{A}<\sum_{q ! N \leqslant(\log N)^{2 A}}^{\sum} \frac{\mu^{2}(q)}{q}
$$

and this is easily seen (by (5) and Lemma) to be $>$ ${ }^{*}{ }_{A} \log \log$ N. The proof is completed by a suitably large choice of $A^{\prime}$ (in terms of $c^{*}$ ) via (3) and (6).

\section{References}

[11 R. Balasubrammian and C. J. Mozzochi, Siegel zeros and the Goldbach Problem, J. Number Theory, 16 (1983), 311-332.

[2] S. Sriaivasan, A Remark on Goldbach Problem, J. Number Theory, 12 (1980), 116-121.

School of Mathematics

Tata Institute of Fundamental Research

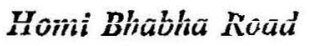

Bombay 400005 (India) 